\title{
Estructura y Representatividad Genética Cloroplástica en Poblaciones Naturales de Anadenanthera colubrina Var. CEBIL (Leguminosae) de Argentina
}

\author{
ALEJANDRA L. GONCALVES ${ }^{1,2,3}$, MARÍA E. BARRANDEGUYY1,2,3 y MARÍA V. GARCÍA ${ }^{1,2,3}$
}

\begin{abstract}
Summary: Chloroplast genetic structure and differentiation in natural populations of Anadenanthera colubrina var. cebil (Leguminosae) from Argentina. Anadenanthera colubrina var. cebil is a native tree from South America distributed in Paranaense and Yungas phytogeographic provinces in Argentina. Individuals from four experimental sites were studied analyzing four cpSSRs loci. Levels of genetic diversity were estimated. Also, population genetic structure was investigated and differentiation of chloroplast genetic variability was estimated. Distribution patterns of chloroplast genetic diversity in the studied populations were consequences of genetic drift. The absence of seed-mediated gene flow between populations and putative historical expansion of Seasonally Dry Tropical Forest followed by fragmentation would have involved in the level of population genetic structure detected. The sampling sites from Paranaense Phytogeographic Province were the less differentiated of the total genetic variation.
\end{abstract}

Key words: Anadenanthera colubrina var. cebil, cpSSR, natural populations.

\begin{abstract}
Resumen: Anadenanthera colubrina var. cebil es una especie forestal nativa de Sudamérica cuya distribución en Argentina se restringe a las provincias fitogeográficas Paranaense y de las Yungas. Se estudiaron individuos pertenecientes a cuatro sitios experimentales mediante el análisis de cuatro loci cpSSRs. Se estimaron los niveles de diversidad genética, se determinó la estructura genética poblacional y se estimó la representatividad de la variabilidad genética cloroplástica. Los patrones de distribución de la diversidad genética cloroplástica contemporáneos en las poblaciones estudiadas resultan de la acción de la deriva genética. La ausencia de flujo génico mediado por semillas entre las poblaciones y la posible expansión histórica de los Bosques Secos Estacionales Neotropicales seguida de fragmentación habrían influido en la marcada estructuración genética poblacional detectada. Los sitios de muestreo de la provincia fitogeográfica Paranaense resultaron los más representativos de la variación genética total.
\end{abstract}

Palabras clave: Anadenanthera colubrina var. cebil, cpSSR, poblaciones naturales.

\section{INTRODUCCIÓN}

El análisis de la distribución y de la representatividad de la variabilidad genética cloroplástica en poblaciones naturales es relevante para el estudio de los procesos demográficos y

\footnotetext{
1 Departamento de Genética. Facultad de Ciencias Exactas, Químicas y Naturales. Universidad Nacional de Misiones, Félix de Azara 1552, (3300) Posadas, Misiones, Argentina. Autor para correspondencia: A. L. Goncalves E-mail: alej.gonc@gmail.com, +54-3764437023 int: 167.

${ }^{2}$ Instituto de Biología Subtropical (UNaM-CONICET).

3 Consejo Nacional de Investigaciones Científicas y Técnicas (CONICET).
}

microevolutivos que actúan de forma concertada para modelar la diversidad genética a través del tiempo y del espacio.

Las especies forestales se distribuyen en ambientes heterogéneos de dimensiones variables (Pastorino \& Gallo, 2009). En caso de interrumpirse la continuidad en su distribución se produce una subdivisión, siendo casi inevitable la diferenciación genética entre los fragmentos cuyas frecuencias alélicas se tornan diferentes. Estos fragmentos, habitualmente son llamados poblaciones locales o subpoblaciones y constituyen las unidades de estudio en Genética de Poblaciones (Hartl \& Clark, 2007).

Anadenanthera colubrina (Vell.) Brenan var. cebil (Griseb.) Altschul (Cialdella, 2000; Tortorelli, 2009), comúnmente conocida en Argentina como "curupay" 
o "cebil colorado" (Tortorelli, 2009), es una especie forestal nativa de América del Sur perteneciente a la subfamilia Mimosoideae y tribu Mimoseae (Cialdella, 2000). Puede alcanzar $35 \mathrm{~m}$ de altura y posee un tronco con corteza grisácea mamelonada (Altschul, 1964). Hojas compuestas bipinnadas con una glándula alargada en la base del pecíolo y 1-3 glándulas similares más pequeñas bajo los últimos pares de pinnas. Las glándulas exudan una sustancia atractiva para insectos polinizadores (Altschul, 1964). Las flores hermafroditas, de color blanco amarillento, se disponen en inflorescencias. El fruto es un folículo largo, angosto y achatado (Queiroz, 2009) con semillas discoidales y aplanadas de color café-negruzco de rápida germinación (Altschul, 1964; Justiniano \& Fredericksen, 1998). Esta especie es alógama (Ciadella, 2000) y la floración ocurre al final de la época seca e inicio de la húmeda; la polinización es entomófila y la diseminación de semillas es por autocoria-anemocoria (Justiniano \& Fredericksen, 1998; Abraham de Noir et al., 2002).

A. colubrina var. cebil se distribuye a lo largo de los Bosques Secos Estacionales Neotropicales (Seasonally Dry Tropical Forests - SDTFs) (Prado \& Gibbs, 1993; Prado, 2000). Prado \& Gibbs (1993) en el marco de la teoría del Arco Pleistocénico propusieron, en base a la semejanza florística de estos bosques, que los patrones actuales de distribución fragmentaria y disyunta de los SDTFs en América del Sur son vestigios de una formación histórica más extensa y continua. A. colubrina var. cebil es considerada la especie más paradigmática de estos bosques (Prado, 2000), pudiendo definirse tres núcleos en su distribución: 1) el núcleo Caatinga, en el NE de Brasil, donde su presencia es muy frecuente, 2) el núcleo Misiones, que ocurre a lo largo de los sistemas de los ríos Paraguay y Paraná en el NE de Argentina, E de Paraguay y SE de Brasil y 3) el núcleo Pedemonte Subandino, en el SO de Bolivia y NO de Argentina (Prado \& Gibbs, 1993) (Fig. 1). En Argentina, la distribución geográfica de esta especie se restringe al Norte del país, presentando una distribución disyunta (Cialdella, 2000) quedando comprendida en las provincias fitogeográficas Paranaense y de las Yungas (Cabrera, 1976).

Para el estudio de la dinámica espacio-temporal de las poblaciones de especies arbóreas, los genomas citoplasmáticos son ampliamente utilizados (Petit \& Vendramin, 2007). El ADN cloroplástico (ADNcp) posee una organización estructural conservada en

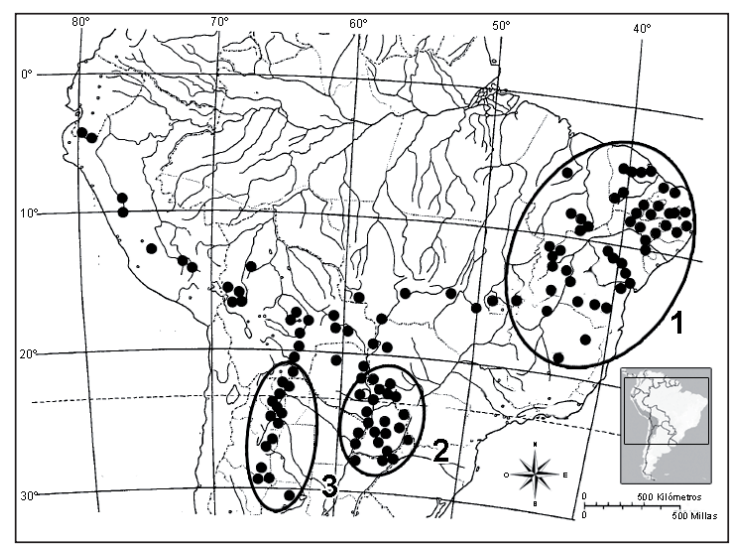

Fig. 1. Distribución de registros de Anadenanthera colubrina var. cebil en Sudamérica. Se indican los núcleos Caatinga (1), Misiones (2) y Pedemonte Subandino (3). Imagen adaptada desde Prado y Gibbs (1993).

las plantas superiores (Ravi et al., 2008), presenta una evolución lenta (Olmstead \& Palmer, 1994) y carece de recombinación (Palmer et al., 1987). Debido a estas características estructurales, es posible interpretar los patrones de variación observados en los diferentes loci en conjunto y asignar haplotipos particulares a los diferentes individuos (Powell et al., 1996). En la mayoría de las Angiospermas, el ADNcp presenta herencia uniparental usualmente por vía materna (Corriveau \& Coleman, 1988) con lo cual puede asumirse que el genoma cloroplástico de $A$. colubrina var. cebil es heredado de manera uniparental por esa vía.

En las plantas superiores, los niveles de estructuración genética de las poblaciones son definidos según el modo de transmisión de sus genomas. En la mayoría de las Angiospermas, al ser las organelas heredadas vía materna, el flujo génico ocurre sólo por semillas (Corriveau \& Coleman, 1988; Palmer et al., 1988). Así, en comparación con los genomas de herencia biparental y paterna, los marcadores heredados por vía materna detectan una mayor diferenciación genética entre las poblaciones (Finkeldey \& Hattemer, 2007) debido a que normalmente las semillas se distribuyen a distancias más cortas que el polen (Zhan et al., 2011).

Los marcadores microsatélites (Simple Sequence Repeat, SSR) son altamente informativos $\mathrm{y}$, probablemente, uno de los marcadores de mayor poder en la detección de polimorfismos. Los loci SSRs se encuentran tanto en el genoma nuclear como 


\section{A. L. Goncalves et al. - Estructura genética cloroplástica de Anadenanthera colubrina var. cebil}

en los genomas citoplasmáticos (Tautz \& Renz, 1984). El estudio de la variación genética cloroplástica se ve facilitado ya que pueden amplificarse regiones SSR mediante cebadores universales complementarios a las regiones del ADNcp conservado que las flanquean (Weising \& Gardner, 1999). Los polimorfismos dados por la variación en el número de repeticiones de los microsatélites cloroplásticos (cpSSR) proporcionan información útil para el estudio de los procesos microevolutivos que generan diferenciación genética y que modelan la composición del acervo génico de las poblaciones a través del tiempo (Powell et al., 1996; Templeton, 2006). El modo de herencia uniparental (Powell et al., 1996), la baja tasa de mutación (Clegg et al., 1994; Provan et al., 1999), la haploidía (Wolfe et al.; 1987; Ennos, 1994) y la ausencia de recombinación (Palmer et al., 1987) del genoma cloroplástico hacen de los marcadores cpSSR una herramienta valiosa para el estudio de los efectos de la fragmentación histórica de las poblaciones, permitiendo analizar el efecto relativo de la acción de la deriva genética y del flujo génico histórico mediado por semillas.

Dada la distribución disyunta de A colubrina var. cebil se analizaron individuos provenientes de cuatro poblaciones naturales del Norte argentino ubicadas en las dos provincias fitogeográficas que abarca su distribución con el objeto de: a) cuantificar la diversidad genética cloroplástica, tanto alélica como haplotípica, b) establecer las relaciones entre los haplotipos a partir de las distancias genéticas, c) analizar la distribución de la variación en los diferentes niveles jerárquicos de organización poblacional, d) determinar la estructura genética cloroplástica de las poblaciones y e) estimar la representatividad de la variabilidad genética cloroplástica en las mismas.

\section{Materiales y Métodos}

\section{Material estudiado}

Se analizó material vegetativo de 69 individuos adultos de A. colubrina var. cebil, de los cuales 36 provenían de la provincia fitogeográfica Paranaense y 33 de la provincia fitogeográfica de las Yungas. De cada individuo se colectaron hojas jóvenes las cuales se conservaron en sílica gel dentro de bolsas de plástico debidamente rotuladas hasta ser procesadas en el laboratorio. Los individuos fueron geo-referenciados mediante el sistema de posicionamiento global (GPS).

En la provincia fitogeográfica Paranaense se consideraron los sitios experimentales Candelaria y Santa Ana, ambos localizados al Sur de Misiones mientras que en las Yungas se consideraron los sitios experimentales Tucumán y Jujuy localizados en la Reserva Horco Molle y en el Parque Nacional Calilegua, respectivamente (Tabla 1; Fig. 2A). Los individuos se muestrearon desde dos sitios en cada uno de ellos, definiendo los sitios de muestreo denominados Candelaria A, Candelaria B, Santa Ana C, Santa Ana D, Tucumán E, Tucumán F, Jujuy G y Jujuy H. Los sitios de muestreo se definieron atendiendo a la distribución fragmentada de esta especie. En la provincia fitogeográfica Paranaense la distancia geográfica promedio entre los sitios de muestreo fue de aproximadamente $1 \mathrm{Km}$ mientras que en las Yungas fue de aproximadamente $4 \mathrm{Km}$ (Tabla 1).

\section{Tabla 1. Localización geográfica y número de individuos en los sitios de A. colubrina var cebil estudiados.}

$\mathrm{N}$ : Número de individuos muestreados.

\begin{tabular}{|c|c|c|c|c|c|c|}
\hline Provincia fitogeográfica & Sitio experimental & Sitio de muestreo & Latitud & Longitud & Altitud (msnm) & $\mathrm{N}$ \\
\hline \multirow{4}{*}{ Paranaense } & \multirow{2}{*}{ Candelaria } & Candelaria A & $27^{\circ} 26^{\prime} 58,20^{\prime \prime} \mathrm{S}$ & $55^{\circ} 44^{\prime} 20,18^{\prime \prime} \mathrm{W}$ & 112 & 10 \\
\hline & & Candelaria B & $27^{\circ} 26^{\prime} 59,89^{\prime \prime} \mathrm{S}$ & $55^{\circ} 44^{\prime} 26,02^{\prime \prime} \mathrm{W}$ & 104 & 10 \\
\hline & \multirow{2}{*}{ Santa Ana } & Santa Ana C & $27^{\circ} 25^{\prime} 55,99^{\prime \prime} \mathrm{S}$ & $55^{\circ} 34^{\prime} 16,79^{\prime \prime} \mathrm{W}$ & 153 & 10 \\
\hline & & Santa Ana D & $27^{\circ} 25^{\prime} 56,39^{\prime \prime} \mathrm{S}$ & $55^{\circ} 34^{\prime} 20,50^{\prime \prime} \mathrm{W}$ & 152 & 6 \\
\hline \multirow{4}{*}{ Yungas } & \multirow{2}{*}{ Tucumán } & Tucumán E & $26^{\circ} 47^{\prime} 26,10^{\prime \prime} \mathrm{S}$ & $65^{\circ} 18^{\prime} 58,14^{\prime \prime} \mathrm{W}$ & 590 & 5 \\
\hline & & Tucumán F & $26^{\circ} 46^{\prime} 32,41^{\prime \prime} \mathrm{S}$ & $65^{\circ} 20^{\prime} 32.21^{\prime \prime} \mathrm{W}$ & 858 & 9 \\
\hline & \multirow{2}{*}{ Jujuy } & Jujuy G & $23^{\circ} 45^{\prime} 15,01^{\prime \prime} \mathrm{S}$ & $64^{\circ} 51^{\prime} 12,99^{\prime \prime} \mathrm{W}$ & 709 & 10 \\
\hline & & Jujuy $\mathrm{H}$ & $23^{\circ} 41^{\prime} 58,70^{\prime \prime} \mathrm{S}$ & $64^{\circ} 51^{\prime} 35.39^{\prime \prime} \mathrm{W}$ & 1026 & 9 \\
\hline
\end{tabular}



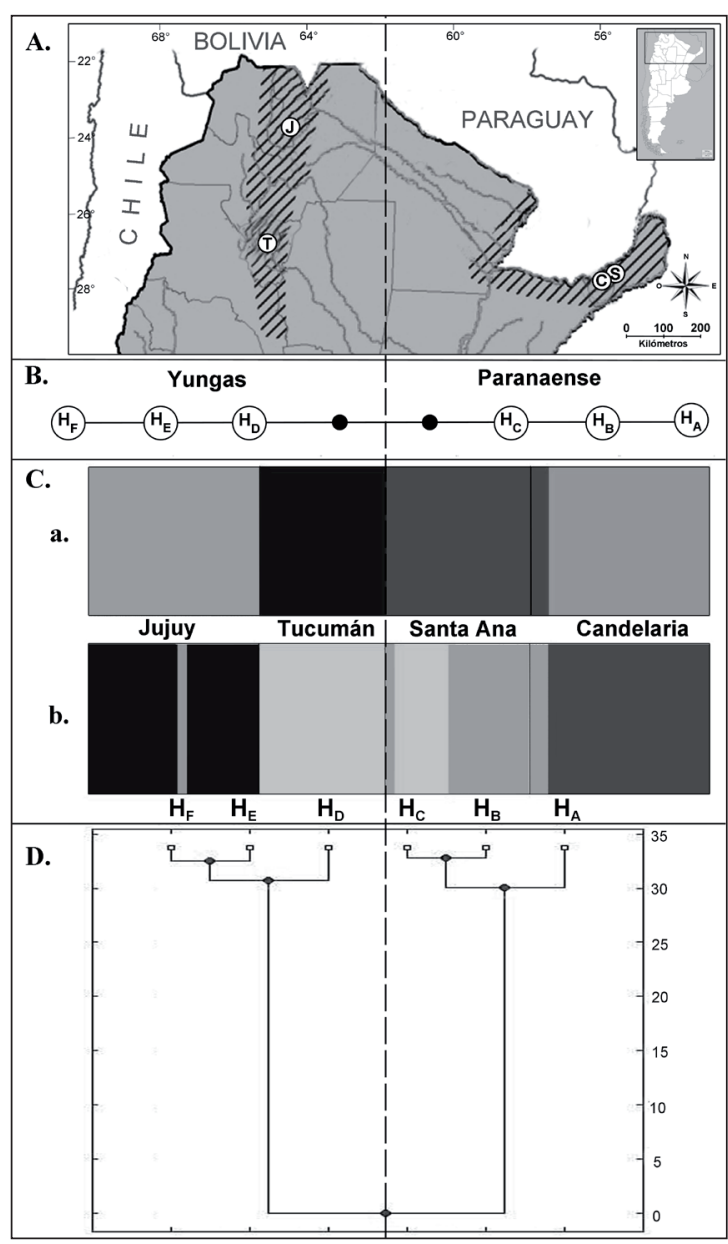

Fig. 2. Análisis de la estructura genética de los sitios experimentales de A. colubrina var. cebil del Norte argentino estudiados. A. Ubicación geográfica de los sitios experimentales: Candelaria (C), Santa Ana $(\mathrm{S})$, Tucumán $(\mathrm{T})$ y Jujuy $(\mathrm{J})$. Se resaltan las áreas de distribución de la especie propuesta por Dimitri et al. (2000); B. Red de haplotipos; C. Análisis Bayesiano: a. Modelo espacial y b. modelo no espacial; D. Dendrograma de los clusters definidos.

\section{Genotipificación mediante marcadores cpSSR}

El ADN genómico total fue extraído empleando el kit de extracción DNeasy ${ }^{\circledR}$ - Plant minikit (Qiagen). Se amplificaron cuatro loci microsatélites cloroplásticos Ccmp2, Ccmp4, Ccmp5 y $C \mathrm{cmp} 7$ mediante cebadores universales desarrollados por Weising \& Gardner (1999), los cuales fueron sintetizados incorporando una marca fluorescente (FAM: azul o HEX: verde) a su extremo 5', para su posterior genotipificación.
Las reacciones de amplificación se realizaron en un volumen final de $15 \mu \mathrm{l}$ empleando $0,5 \mathrm{ng} /$ $\mu \mathrm{l}$ de ADN, 1x de Buffer Hot Start, 2,5 mM de $\mathrm{Cl}_{2} \mathrm{Mg}, 0,2 \mathrm{mM}$ de cada dNTP, $1 \mathrm{U}$ de enzima ADN polimerasa Hot Start (5 U/ $\mu$ l FirePol Solis BioDyne) y 0,33 pmol de cada cebador. La reacción de amplificación fue realizada en un termociclador con gradiente (Biometra) empleando un programa de tipo touchdown. Las condiciones de ciclado empleadas fueron: una desnaturalización inicial a $94^{\circ} \mathrm{C}$ durante $15 \mathrm{~min}$, seguida de 10 ciclos de: desnaturalización a $94^{\circ} \mathrm{C}$ durante $1 \mathrm{~min}$, hibridación en un rango de temperatura de 60 a $50^{\circ} \mathrm{C}$ durante 1 min $\left(-1^{\circ} \mathrm{C}\right.$ por ciclo) y elongación a $72^{\circ} \mathrm{C}$ durante 1 min. Estos 10 ciclos fueron seguidos por 29 ciclos de: desnaturalización a $94^{\circ} \mathrm{C}$ durante $1 \mathrm{~min}$, hibridación a $50^{\circ} \mathrm{C}$ durante 1 min y elongación a $72^{\circ} \mathrm{C}$ durante $1 \mathrm{~min}$. La reacción de amplificación terminó con una etapa de elongación final a $72^{\circ} \mathrm{C}$ durante $20 \mathrm{~min}$.

Los diferentes alelos fueron identificados a partir del tamaño de los fragmentos el cual fue asignado empleando el secuenciador ABI Prism ${ }^{\circledR}$ 3100 (Applied Biosystems) mediante el empleo del programa GENESCAN ${ }^{\mathrm{TM}}$ y un marcador de peso estándar GS $500 \mathrm{ROX}^{\mathrm{TM}}$ (Applied Biosystems). Mediante la combinación de los alelos de los diferentes loci se definieron los haplotipos.

\section{Análisis estadístico de los datos}

Las estimaciones de diversidad genética fueron realizadas para cada sitio experimental y de manera global. El número promedio de alelos por locus $(\mathrm{Na})$ y el número promedio de alelos únicos (AU), se estimaron mediante el programa GenAlEx 6.5 (Peakall \& Smouse, 2012). El número de haplotipos $(\mathrm{NH})$, el número de haplotipos únicos (HU) y el número de haplotipos compartidos (HC) se determinaron por conteo directo, en tanto que el índice de diversidad haplotípica de Nei $h$ (Nei, 1987) fue obtenido mediante cálculo manual.

La distribución de la variación haplotípica dentro y entre los sitios experimentales, se analizó por medio de un Análisis de Varianza Molecular (AMOVA) testando la hipótesis nula de ausencia de estructuración genética (Stewart \& Excoffier, 1996). El AMOVA partió de una matriz de distancias cuadráticas construida entre todos los pares de haplotipos aplicando el índice métrico Euclideano (Huff et al., 1993). El modelo jerárquico 
incluyó como fuentes de variación a los siguientes niveles: "dentro de sitios experimentales", "entre sitios experimentales dentro de cada provincia fitogeográfica”, y “entre provincias fitogeográficas". La significancia estadística de los componentes de la covarianza asociados a estos niveles de estructura genética, fue testada bajo un enfoque no paramétrico basado en permutaciones ya que los datos moleculares no presentan una distribución normal (Excoffier et al., 1992).

El grado de estructuración genética poblacional se analizó mediante el índice de fijación $\mathrm{F}_{\mathrm{ST}}$ (Wright, 1965), el cual fue estimado a partir del AMOVA. La significancia estadística de este índice fue testada con un valor de $p$ obtenido mediante 1023 permutaciones de los haplotipos dentro de sitios experimentales y entre provincias fitogeográficas para el $95 \%$ de confianza $(p<0,05)$. Estos análisis fueron desarrollados utilizando el programa Arlequin 3.5 (Excoffier \& Lischer, 2010).

Las relaciones entre los haplotipos se infirieron mediante la construcción de una red mínima (minimum spanning network) graficando las distancias genéticas a partir del número de alelos diferentes entre todos los pares de haplotipos. La red fue graficada por medio del programa HapStar 0.7 (Teacher \& Griffiths, 2011).

Se infirió la estructura genética poblacional mediante un análisis bayesiano usando un modelo de agrupamiento de tipo mixture (Non-spatial genetic mixture analysis) para loci ligados (Corander et al., 2008a). Mediante este análisis se determinó el número más probable de agrupamientos o clusters y se asignaron los individuos a ellos empleando únicamente información genética. El número de clusters fue tratado como un parámetro desconocido. Además, se implementó un modelo de agrupamiento espacial de individuos (Spatialgenetic mixture analysis) para loci ligados (Corander et al., 2008b) incorporando al agrupamiento no espacial el término correspondiente a la probabilidad a priori de la posición geográfica de cada individuo. Para determinar el número de clusters que presenta la mayor probabilidad a posteriori se testaron valores de $k$ clusters comprendidos en un rango de dos a ocho $(k=[2-8])$ realizando 10 repeticiones para cada valor de $k$.

Las relaciones existentes entre los clusters fueron determinadas mediante la construcción de un dendrograma aplicando la técnica de agrupamiento
UPGMA (Unweighted Pair Groups Method with Arithmetic Mean) a la matriz de distancia obtenida a partir de los clusters. Esta matriz se obtuvo aplicando el índice de distancia de Kullback-Leibler (KL) (Kullback \& Leibler, 1951) el cual puede emplearse como una medida de distancia genética relativa entre la distribución de dos conjuntos de datos (Kasturi et al., 2003). La totalidad de estos análisis fueron desarrollados utilizando el programa BAPS versión 6.0 (Corander et al., 2006, 2008a).

Se determinó la representatividad de la variabilidad genética cloroplástica de cada sitio de muestreo por la diferencia entre su composición genética y la de su complemento mediante la estimación del índice de diferenciación genética de Gregorius $D_{j}$ (Gregorius, 1984). Además, se estimó el índice de diferenciación subpoblacional $(\delta)$ para representar el nivel medio de diferenciación entre los sitios de muestreo (Gregorius \& Roberds, 1986). De esta manera, se caracterizó a cada sitio de muestreo por la diferencia entre su composición genética y la de su complemento considerando dos niveles de análisis: a nivel de acervo génico, en el cual los elementos genéticos correspondieron a todos los alelos y a nivel de haplotipos, en el cual los elementos genéticos correspondieron a la combinación de alelos para los diferentes loci. La totalidad de estos análisis fueron realizados utilizando el programa GSED versión 3.0 (Gillet, 2010).

\section{Resultados}

A partir de los cuatro loci analizados se definieron seis haplotipos $\left(\mathrm{H}_{\mathrm{A}}-\mathrm{H}_{\mathrm{F}}\right)$. El locus Ccmp5 fue el más polimórfico debido a que presentó cuatro alelos diferentes. El alelo 109 del locus Ccmp 4 estuvo presente tanto en individuos de las Yungas como en individuos de Santa Ana (Tabla 2). Los sitios experimentales analizados presentaron baja diversidad genética en todos los loci cpSSRs analizados. En los 69 individuos se detectó, en promedio, un alelo por locus $(\mathrm{Na})$ y menos de un alelo único por locus (AU) en todos los sitios experimentales, registrándose ausencia de alelos únicos en Santa Ana mientras que Jujuy presentó la mayor cantidad de alelos únicos (Tabla 3). Se definieron seis haplotipos, de los cuales uno $\left(\mathrm{H}_{\mathrm{B}}\right)$ fue compartido entre Candelaria y Santa Ana, en tanto que los haplotipos restantes $\left(\mathrm{H}_{\mathrm{A}}, \mathrm{H}_{\mathrm{C}}, \mathrm{H}_{\mathrm{D}}, \mathrm{H}_{\mathrm{E}}\right.$ 
Bol. Soc. Argent. Bot. 49 (2) 2014

Tabla 2. Haplotipos cpSSRs analizados en A. colubrina var. cebil. N: número de individuos

Se indican los alelos según su tamaño en pares de bases.

\begin{tabular}{|ccccccc|}
\hline Haplotipo & Ccmp2 & Ccmp4 & Ccmp5 & Ccmp7 & Sitio de muestreo & N \\
\hline $\mathbf{H}_{\mathrm{A}}$ & 478 & 112 & 133 & 155 & Candelaria A y B & 18 \\
$\mathbf{H}_{\mathrm{B}}$ & 478 & 112 & 134 & 155 & Candelaria B; Santa Ana C y D & 12 \\
$\mathbf{H}_{\mathrm{C}}$ & 478 & 109 & 134 & 155 & Santa Ana D & 6 \\
$\mathbf{H}_{\mathrm{D}}$ & 491 & 109 & 140 & 150 & Tucumán E y F & 14 \\
$\mathbf{H}_{\mathrm{E}}$ & 474 & 109 & 140 & 150 & Jujuy G y H & 18 \\
$\mathbf{H}_{\mathrm{F}}$ & 474 & 109 & 139 & 150 & Jujuy G & 1 \\
\hline
\end{tabular}

$\mathrm{y}_{\mathrm{F}}$ ) fueron únicos, es decir, cada haplotipo fue propio de un sitio experimental. Estos resultados denotan ausencia de haplotipos compartidos entre las provincia fitogeográficas (Tablas 2, 3). El índice de diversidad haplotípica de Nei (h), presentó un valor promedio bajo siendo moderado en los sitios experimentales de la provincia fitogeográfica Paranaense y bajo en los de las Yungas (Tabla 3).

En relación al análisis de la distribución de la variación molecular en los diferentes niveles jerárquicos de organización, el AMOVA indicó que el mayor porcentaje de variación estuvo contenido entre las provincias fitogeográficas $(69,69 \%)$. Entre sitios experimentales dentro de provincias fitogeográficas se resumió el 25,11\% y dentro de los sitios experimentales se observó el menor porcentaje de variación $(5,21 \%)$ (Tabla 4). El índice de fijación $\mathrm{F}_{\mathrm{ST}}$ indicó una fuerte estructuración genética debido a que alcanzó un valor de 0,95 resultando estadísticamente significativo a un nivel del 95\% de confianza $(p<0,05)$.
La red de haplotipos evidenció mayor distancia genética entre haplotipos provenientes de las diferentes provincias fitogeográficas en relación a la distancia genética presente entre haplotipos de cada provincia (Fig. 2B).

El modelo no espacial del análisis Bayesiano estableció que el número más probable de clusters fue seis $\left(\mathrm{P}_{(\mathrm{k}=6)}=0,80\right)$, presentando los individuos asignados a cada cluster el mismo haplotipo. Por su parte, el modelo espacial estableció que el número más probable de clusters fue cuatro $\left(\mathrm{P}_{(\mathrm{k}=4)}=0,99\right)$, presentando los individuos asignados a cada cluster el mismo origen geográfico, a excepción de dos individuos de Candelaria los cuales portan el único haplotipo compartido (Fig. 2.C). A partir de la definición de estos cuatro clusters, los sitios experimentales pueden ser considerados poblaciones desde un punto de vista genético, es decir un conjunto de individuos que comparten un acervo génico característico (Hartl \& Clark, 2007).

Tabla 3. Diversidad genética en los sitios experimentales de A. colubrina var. cebil estudiados. N: Número de individuos muestreados, Na: Número promedio de alelos por locus, AU: Número promedio de alelos únicos, NH: Número de haplotipos, HU: Número de haplotipos únicos, HC: Número de haplotipos compartidos y h: Indice de diversidad haplotípica de Nei. *Valor promedio entre sitios experimentales.

\begin{tabular}{|c|c|c|c|c|c|c|c|c|}
\hline Provincia fitogeográfica & Sitio experimental & $\mathbf{N}$ & $\mathrm{Na}$ & $\mathrm{AU}$ & NH & HU & $\mathrm{HC}$ & $\mathbf{h}$ \\
\hline \multirow{2}{*}{ Paranaense } & Candelaria & 20 & 1,250 & 0,250 & 2 & 1 & 1 & 0,400 \\
\hline & Santa Ana & 16 & 1,250 & 0,000 & 2 & 1 & 1 & 0,500 \\
\hline \multirow{2}{*}{ Yungas } & Tucumán & 14 & 1,000 & 0,250 & 1 & 1 & 0 & 0,000 \\
\hline & Jujuy & 19 & 1,250 & 0,500 & 2 & 2 & 0 & 0,094 \\
\hline Total & & 69 & $1,188^{*}$ & $0,250^{*}$ & 6 & 5 & 1 & $0,249^{*}$ \\
\hline
\end{tabular}


Tabla 4. Análisis de Varianza Molecular.

\begin{tabular}{|ccccc|}
\hline Fuente de Variación & $\begin{array}{c}\text { Grados de } \\
\text { libertad }\end{array}$ & $\begin{array}{c}\text { Suma de } \\
\text { cuadrados }\end{array}$ & $\begin{array}{c}\text { Componentes } \\
\text { de varianza }\end{array}$ & $\begin{array}{c}\text { Porcentaje de } \\
\text { variación }\end{array}$ \\
\hline Entre provincias fitogeográficas & 1 & 54,59 & $1,34 \mathrm{Va}$ & $\mathbf{6 9 , 6 9 \%}$ \\
$\begin{array}{c}\text { Entre sitios experimentales, dentro } \\
\text { de provincias fitogeográficas }\end{array}$ & 2 & 16,53 & $0,48 \mathrm{Vb}$ & $\mathbf{2 5 , 1 1 \%}$ \\
\hline Dentro de sitios experimentales & 65 & 6,5 & $0,10 \mathrm{Vc}$ & $\mathbf{5 , 2 1 \%}$ \\
\hline Total & 68 & 77,62 & 1,92 & $100 \%$ \\
\hline
\end{tabular}

El dendrograma agrupó los seis clusters definidos mediante el modelo no espacial del análisis Bayesiano en dos grupos principales, en coincidencia con la provincia fitogeográfica de origen de los individuos asignados a los mismos. Las distancias genéticas entre los clusters asumen valores similares dentro de cada provincia (Fig. 2D).

Al caracterizar cada sitio de muestreo por la diferencia entre su composición genética y la de su complemento, los niveles de diferenciación reflejados por los valores de $D_{j}$ y de $\delta$ mostraron que la diferenciación genética entre los sitios de muestreo fue mayor a nivel de haplotipos que a nivel de acervo génico (Fig. 3). Los sitios de muestreo Tucumán $\mathrm{E}$ y Tucumán $\mathrm{F}$ fueron analizados como una única entidad debido a que presentaron idéntica composición genética. En este último análisis, el sitio experimental Tucumán presentó mayor diferenciación genética respecto a su complemento tanto a nivel de haplotipos como de acervo génico. Por su parte, el sitio de muestreo Candelaria B presentó mayor representatividad a nivel de haplotipos, puesto que posee dos haplotipos compartidos a elevada frecuencia, en tanto que el sitio de muestreo Santa Ana D presentó mayor representatividad a nivel de acervo génico debido a que comparte los dos alelos del locus Ccmp4 con todos los sitios de muestreo. De esta manera, a nivel de acervo génico los sitios de muestreo de la provincia fitogeográfica Paranaense fueron los más representativos de la variación genética total mientras que los de las Yungas fueron los más diferenciados (Fig. 3).

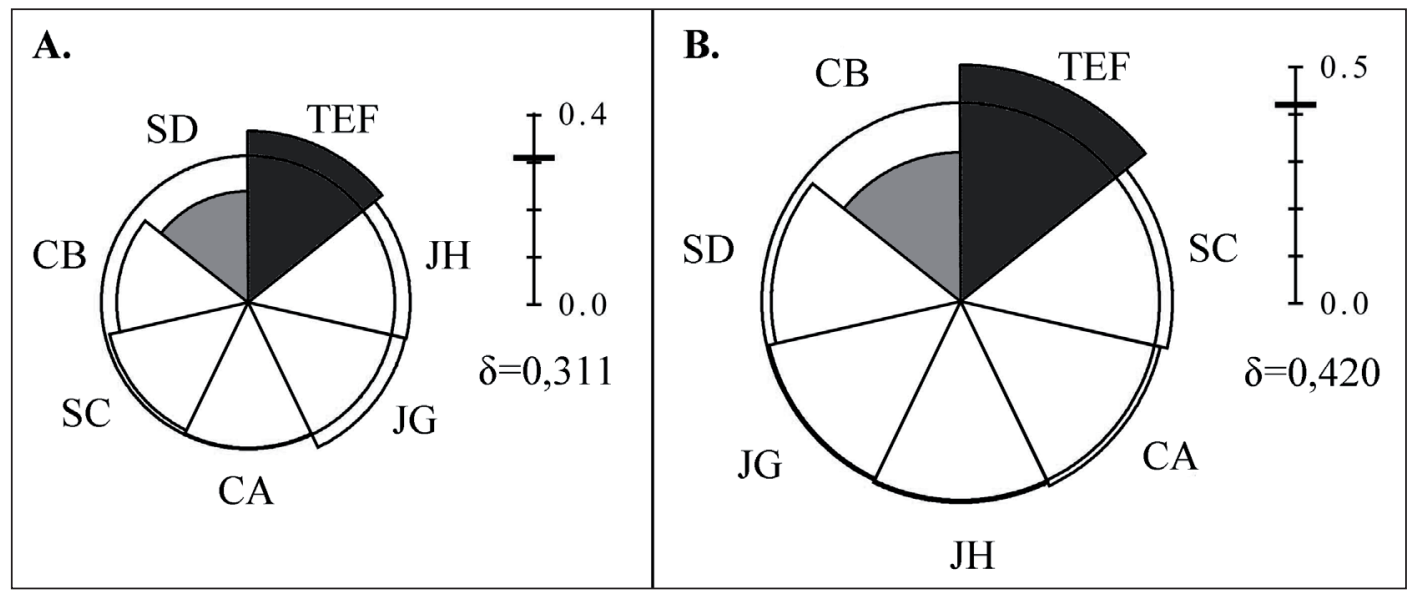

Fig. 3. Análisis de la representatividad de la variabilidad genética cloroplástica a nivel de A. acervo génico y B. haplotipos. El radio de cada porción es proporcional a Dj. El radio del círculo es proporcional a $\delta$. Sitios de muestreo: CA: Candelaria A; CB: Candelaria B, SC: Santa Ana C; SD: Santa Ana D; TEF: Tucumán; JG: Jujuy G; JH: Jujuy H. Se indica $\square$ : sitio de muestreo más diferenciado y $\square$ : sitio de muestreo más representativo. 


\section{Discusión}

La distribución de la variabilidad genética cloroplástica resulta de la acción conjunta de los procesos microevolutivos, tales como deriva genética y flujo génico, y de los eventos demográficos, los cuales han operado en tiempos pasados y se encuentran operando en el presente modelando esta variabilidad genética.

Es posible realizar inferencias sobre la acción de los diferentes procesos microevolutivos por medio del análisis de la diversidad genética en los loci cpSSRs y la determinación del grado de estructuración genética poblacional (Provan et al., 1999).

Los bajos niveles de polimorfismo en estos loci, reflejados en la baja diversidad genética y haplotípica promedio detectada en los sitios experimentales de la provincia fitogeográfica Paranaense pueden esperarse debido a que la tasa de mutación de los cpSSRs es baja, siendo en promedio $1 \times 10^{-5}$ (Vachon \& Freeland, 2011). A pesar de ello, estos marcadores resultaron eficaces para detectar variación entre sitios experimentales y entre provincias fitogeográficas, presentando tres de los cuatro sitios experimentales haplotipos únicos y ausencia de haplotipos compartidos entre las provincias fitogeográficas. Esta distribución haplotípica se pone de manifiesto en la marcada estructuración genética poblacional revelada por el índice de fijación $\left(\mathrm{F}_{\mathrm{ST}}=0,95\right)$ ya que un valor de $\mathrm{F}_{\mathrm{ST}}$ cercano a uno indica la fijación de diferentes haplotipos en las diferentes poblaciones (Wright, 1965).

Los niveles de dispersión de semillas pueden modelar la composición y la estructura genética de las poblaciones vegetales (Hamrick et al., 1993). Ha sido sugerido que las especies que producen semillas con bajo potencial de dispersión generalmente poseen menor diversidad genética y elevados niveles de estructuración genética poblacional en el ADNcp (Pakkad et al., 2008). El mecanismo de dispersión de A. colubrina var. cebil es autocórico-anemocórico (Abraham de Noir et al., 2002) presentando sus semillas un bajo potencial de dispersión y en coincidencia con lo sugerido, se observó baja diversidad y elevada estructuración genética en los sitios experimentales estudiados. Los niveles de estructuración genética presentes en los sitios experimentales de A. colubrina var. cebil son similares a los detectados por Lira et al. (2003) en Caesalpinia echinata Lam. $\left(\mathrm{F}_{\mathrm{ST}}=0,95\right.$ y $\mathrm{F}_{\mathrm{ST}}=0,91$, respectivamente). Ambas especies presentan dispersión de semillas por gravedad. En contraparte, Eugenia uniflora cuya dispersión de semillas es zoócora presenta menor estructuración $\left(\mathrm{F}_{\mathrm{ST}}=0,21\right)$ (Salgueiro et al., 2004).

Los métodos de inferencia bayesiana permitieron establecer el número y la composición de las poblaciones empleando únicamente criterios genéticos (Corander et al., 2003). Mediante este modelo se identificaron seis clusters, presentando los individuos asignados a cada uno de ellos el mismo haplotipo. Además, estos clusters se agruparon de acuerdo a su región de origen, presentándose la máxima distancia genética entre las provincias fitogeográficas (Fig. 2D) aunque dentro de ambas provincias fitogeográficas las distancias genéticas entre los clusters fueron bajas. En la provincia fitogeográfica Paranaense la cercanía geográfica entre las poblaciones podría explicar la baja distancia genética mientras que, en la provincia de las Yungas las poblaciones analizadas se ubican a una gran distancia geográfica. A pesar de la gran distancia geográfica, las poblaciones Tucumán y Jujuy se encuentran localizadas en el núcleo Pedemonte subandino con lo cual comparten una misma dinámica histórica (Werneck et al., 2011) pudiendo ambas poblaciones presentar una ascendencia común y traducirse esto en una baja distancia genética.

La consideración conjunta del mecanismo de dispersión de semillas de A. colubrina var. cebil y de la distancia geográfica entre las poblaciones, hace poco probable la existencia de flujo génico mediado por semillas entre ellas. Al incorporar al análisis bayesiano la localización geográfica de los individuos como probabilidad a priori, el número de clusters se redujo a cuatro, coincidiendo su composición mayoritariamente con los sitios experimentales de origen reflejando de esta manera, ausencia de flujo génico mediado por semillas. Cuando las poblaciones se aíslan unas de otras, las fuerzas estocásticas remodelan la composición genética de los individuos a lo largo de las generaciones siendo la deriva genética la fuerza microevolutiva responsable de la estructuración genética poblacional (Corander et al., 2008a). Este proceso tiende a la fijación o pérdida de alelos y su naturaleza aleatoria hace poco probable que las poblaciones locales presenten las 
mismas variantes fijadas (Templeton, 2006). Es así que la fijación del haplotipo $\mathrm{H}_{\mathrm{D}}$ en Tucumán, sería resultado de la acción de la deriva genética tras largos períodos de aislamiento.

A partir de la distribución de los haplotipos cloroplásticos en las poblaciones analizadas, conociendo la naturaleza y evolución de este genoma como así también las teorías existentes acerca de los eventos demográficos ocurridos en los SDTFs, los patrones de distribución de la diversidad genética cloroplástica contemporáneos pueden interpretarse como resultado de los eventos demográficos históricos. La posible expansión histórica de los SDTFs seguida de su fragmentación habría jugado un rol preponderante sobre los niveles de estructuración genética de las poblaciones de $A$. colubrina var. cebil estudiadas dado que las mismas presentaron bajos niveles de diversidad genética cloroplástica y elevadas frecuencias de alelos y haplotipos únicos.

Los SDTFs representan uno de los ecosistemas tropicales más amenazados debido a su alta tasa de deforestación (Janzen, 1988; GarcíaOliva \& Jaramillo, 2011). El conocimiento de la representatividad de la variabilidad genética contemporánea es de utilidad en la planificación de estrategias de conservación de los recursos genéticos contribuyendo a la identificación de cuántas y cuáles poblaciones conservar (Gillet et al., 2005; Zhao \& Gong, 2012). En sentido estricto, el término conservación genética implica preservar el número máximo de alelos de un recurso genético (Finkeldey \& Hattemer, 2007; Höglund, 2009). Esta definición enfatiza la necesidad de considerar tanto a las poblaciones más diferenciadas como a las más representativas en planes de conservación. En especies vegetales no cultivadas, incluyendo especies forestales nativas, la preservación del máximo número de alelos deja de ser el objetivo principal debido a que alelos raros o únicos no mejorarían la capacidad de las poblaciones para sobrevivir en un ambiente particular a cambios ambientales repentinos (Finkeldey \& Hattemer, 2007) adquiriendo de esta manera, mayor relevancia la preservación de la variación genética más representativa. Los sitios de muestreo de la provincia fitogeográfica Paranaense presentaron la mayor representatividad de la variación genética total con lo cual estos sitios de muestreo deberían ser considerados en futuros planes de conservación.

\section{Conclusiones}

A. colubrina var. cebil es un recurso nativo de Sudamérica que presenta una distribución disyunta en el Norte argentino. Los patrones de distribución de la diversidad genética cloroplástica contemporáneos en las poblaciones estudiadas, caracterizados por bajos niveles de diversidad genética, presencia de haplotipos únicos y ausencia de haplotipos compartidos entre las provincias fitogeográficas, resultan de la acción de la deriva genética. La ausencia de flujo génico mediado por semillas entre las poblaciones y la posible expansión histórica de los SDTFs seguida de fragmentación habrían influido en la marcada estructuración genética poblacional detectada. Los sitios de muestreo de la provincia fitogeográfica Paranaense resultaron los más representativos de la variación genética total.

\section{Agradecimientos}

Este trabajo fue parcialmente financiado mediante el subsidio PIP-IU 2010-2012 No 114 200901-00110 otorgado a M. V. García por el Consejo Nacional de Investigaciones Científicas y Técnicas (CONICET).

\section{Bibliografía}

ABRAHAM DE NOIR, F., S. BRAVO \& R. ABDALA. 2002. Mecanismos de dispersión de algunas especies de leñosas nativas del chaco occidental y serrano. Quebracho 9: 140-150.

ALTSCHUL, S. VON R. 1964. A taxonomic study of the genus Anadenanthera. Contr. Gray Herb. 193: 3-65.

CABRERA, A. L. 1976. Regiones fitogeográficas Argentinas. En: Kluger W. F. (ed.), Enciclopedia Argentina de Agricultura y Jardinería, 2a Ed., Tomo 2, pp. 1-85. Acme, Buenos Aires.

CIALDELLA, A. M. 2000. Fabaceae Subfamilia Mimosoideae, Flora Fanerogámica Argentina, Fascículo 67: 1-10. Programa Pro Flora-CONICET, Córdoba.

CLEGG, M. T., B. S. GAUT, G. H. LEARN \& B. R. MORTON. 1994. Rates and patterns of chloroplast DNA evolution. Proc. Natl. Acad. Sci. USA. 91: 6795-6801.

CORANDER, J., P. WALDMANN \& M. J. SILLANPÄÄ. 2003. Bayesian analysis of genetic differentiation between populations. Genetics 163: 367-374. 
CORANDER, J., P. MARTTINEN \& S. MÄNTYNIEMI. 2006. Bayesian identification of stock mixtures from molecular marker data. Fish. B. 104: 550-558.

CORANDER, J., P. MARTTINEN, J. SIRÉN \& J. TANG. 2008a. Enhanced Bayesian modelling in BAPS software for learning genetic structures of populations. BMC Bioinformatics 9: 539.

CORANDER, J., J. SIRÉN \& E. ARJAS. 2008b. Bayesian Spatial Modelling of Genetic Population Structure. Comput. Stat. 23: 111-129.

CORRIVEAU, J.L. \& A.W. COLEMAN. 1988. Rapid screening method to detect potential biparental inheritance of plastid DNA and results for over 200 angiosperm species. Amer. J. Bot. 75: 1443-1458.

DIMITRI, M. J., R. F. J. LEONARDIS \& J. S. BILONI. 2000. El nuevo libro del árbol. 4a Ed. Manufacturas Editoriales Litográficas, Madrid.

ENNOS, R.A. 1994. Estimating the relative rates of pollen and seed migration among plant populations. Heredity 72: 250-259.

EXCOFFIER, L., P. E. SMOUSE \& J. QUATTRO. 1992. Analysis of molecular variance inferred from metric distances among DNA haplotypes: Application to human mitochondrial DNA restriction data. Genetics 131: 479-491.

EXCOFFIER, L. \& H. E. L. LISCHER. 2010. Arlequin suite ver 3.5: A new series of programs to perform population genetics analyses under Linux and Windows. Molec. Ecol. Resour. 10: 564-567.

FINKELDEY, R. \& H. H. HATTEMER. 2007. Tropical forest genetics. Springer-Verlag, Berlín-Heidelberg.

GARCÍA-OLIVA, F. \& V. J. JARAMILLO. 2011. Impact of Anthropogenic Transformation of Seasonally Dry Tropical Forests on Ecosystem Biogeochemical Processes. In: DIRZO R., H. S. YOUNG, H. A. MOONEY \& G. CEBALLOS (eds.), Seasonally Dry Tropical Forests: Ecology and Conservation, pp. 159-172. Island Press, Washington DC.

GILLET, E. 2010. GSED Genetic Structures from Electrophoresis Version 3.0 Data User's Manual. Abt. Forstgenetik und Forstpflanzenzüchtung, Universität Göttingen, Göttingen.

GILlET, E., D. GÖMÖRY \& L. PAULE. 2005. Measuring genetic variation within and among populations at marker loci. In: GEBUREK, T. \& J. TUROK (eds.), Conservation and Management of forest genetic resource in Europe, pp 237-274. Arbora Publishers, Zvolen.

GREGORIUS, H. R. 1984. Measurement of genetic differentiation in plant populations. In: GREGORIUS, H.R (ed.), Population Genetics in Forestry, pp. 276-285. Springer-Verlag, Berlín.

GREGORIUS, H. R. \& J. H. ROBERDS. 1986. Measurement of genetical diferentiation among subpopulations. Theor. Appl. Genet. 71: 826-834.
HAMRICK, J. L., MURAWSKI D. A. \& NASON J. D. 1993. The influence of seed dispersal mechanisms on the genetic structure of tropical tree populations. Vegetatio 107(108): 281-297.

HARTL, D. L. \& A. G. CLARK. 2007. Principles of population genetics. 4th ed. Sinauer Associates, Sunderland.

HÖGLUND, J. 2009. Evolutionary Conservation Genetics. Oxford University Press, New York.

HUFF, D. R, R. PEAKALL \& P. E. SMOUSE. 1993. RAPD variation within and among natural populations of outcrossing buffalograss [Buchloë dactyloides (Nutt.) Engelm]. Theor. Appl. Genet. 86:927-934.

JANZEN, D. H. 1988. Tropical Dry forest, the most endangered major tropical ecosystem. In: WILSON, E. O. (ed.), Biodiversity, pp. 130-37. National Academies Press, Washington DC.

JUSTINIANO, M. J. \& T. S. FREDERICKSEN. 1998. Ecología y Silvicultura de especies menos conocidas: Curupaú. Anadenanthera colubrina (Vell.) Benth. Mimosoideae, pp. 1-30. Proyecto de Manejo Forestal Sostenible Bolfor.

KASTURI, J., R. ACHARYA \& M. RAMANATHAN. 2003. An information theoretic approach for analyzing temporal patterns of gene expression. Bioinformatics 19: 449-458.

KULLBACK, S. \& R. A. LEIBLER. 1951. On information and sufficiency. Ann. Math. Stat. 22:79-86.

LIRA, C.F., S.R.S. CARDOSO, P. C. G. FERREIRA, M.A. CARDOSO, \& J. PROVAN. 2003. Long-term population isolation in the endangered tropical tree species Caesalpinia echinata Lam. revealed by chloroplast microsatellites. Molec. Ecol. 12: 32193225 .

NEI, M. 1987. Molecular evolutionary genetics. Columbia University Press, New York.

OLMSTEAD, R. G. \& J. D. PALMER. 1994. Chloroplast DNA Systematics: A Review of Methods and Data Analysis. Amer. J. Bot. 81: 1205-1224.

PAKKAD, G., S. UENO \& H. YOSHIMARU. 2008. Genetic differentiation of Quercus semiserrata Roxb. In northern Thailand revealed by nuclear and chloroplast microsatellite markers. Forest. Ecol. Manag. 255: 1067-1077.

PALMER J.D., B. OSORIO, J. ALDRICH, \& W.F. THOMPSON. 1987. Chloroplast DNA evolution among legumes: Loss of a large inverted repeat occurred prior to other sequence rearrangements. Curr. Genet. 11: 275-286

PALMER, J. D., R. K. JANSEN, H. J. MICHAELS, M. W. CHASE \& J. R. MANHART. 1988. Chloroplast DNA variation and plant phylogeny. Ann. Misouri. Bot. Gard. 75: 1180-1206.

PASTORINO, M. J. \& L. A. GALLO. 2009. Preliminary 
operational genetic management units of a highly fragmented forest tree species of southern South America. Forest Ecol. Manag. 257: 2350-2358.

PEAKALL, R. \& SMOUSE P. E. 2012. GenAlEx 6.5: genetic analysis in Excel. Population genetic software for teaching and research-an update. Bioinformatics 28: 2537-2539.

PETIT, R. J. \& G. G.VENDRAMIN. 2007. Plant phylogeography based on organelle genes: an introduction. In: WEISS S. \& N. FERRAND (eds.), Phylogeography of Southern European Refugia - evolutionary perspective on the origins and conservation of European biodiversity, pp. 23-97. Springer, Berlín.

POWELL, W., G.C. MACHRAY \& J. PROVAN. 1996. Polymorphism revealed by simple sequence repeat. Trends Plant Sci. 1: 215-222.

PRADO, D. 2000. Seasonally dry forest of tropical South America: from forgotten ecosystems to a new phytogeography unit. Edinb. J. Bot. 57: 437-461.

PRADO, D. E. \& P. E. GIBBS. 1993. Patterns of species distributions in the Dry Seasonal Forests of South America. Ann. Missouri Bot. Gard. 80: 902-927.

PROVAN, J., N. SORANZO, N. J. WILSON, D. GOLSTEIN \& W. POWEL. 1999. A low rate for chloroplast microsateéllites. Genetics 153: 943-947.

QUEIROZ, L. 2009. Leguminosas da Caatinga. Univ. Estad. Feira de Santana, RBG, Kew \& Ass Plantas do Nordeste, Recife.

RAVI, V., J.P. KHURANA, A.K. TYAGI \& P. KHURANA. 2008. An update on chloroplast genomes. Plant Syst. Evol. 271: 101-122.

SALGUEIRO, F., D. FELIX, J. F. CALDAS, M. MARGIS-PINHEIRO \& R. MARGIS. 2004. Even population differentiation for maternal and biparental gene markers in Eugenia uniflora, a widely distributed species from the Brazilian coastal Atlantic rain forest. Divers. Distrib. 10: 201-210.

STEWART, C. N. \& L. EXCOFFIER. 1996. Assessing population genetic structure and variability with RAPD data: Application to Vaccinium macrocarpa (American cranberry). J. Evol. Biol. 9: 153-171.

TAUTZ, D. \& M. RENZ. 1984. Simple sequences are ubiquitous repetitive components of eukaryotic genomes. Nucleic Acids Res. 12: 4127-4138.
TEACHER, A. G. F. \& D. J. GRIFFITHS. 2011. HapStar: automated haplotype network layout and visualization. Molec. Ecol. Resour. 11: 151-153.

TEMPLETON, A. R. 2006. Population genetics and microevolutionary theory. Wiley-Liss, Hoboken.

TORTORELLI, L. A. 2009. Maderas y bosques argentinos. Orientación Gráfica Editora, Buenos Aires.

VACHON, N., \& FREELAND, J. R. 2011. Phylogeographic inferences from chloroplast DNA: quantifying the effects of mutations in repetitive and non-repetitive sequences. Molec. Ecol. Resour. 11: 279-85.

WEISING, K. \& R. C. GARDNER. 1999. A set of conserved PCR primers for the analysis of simple sequence repeat polymorphisms in chloroplast genomes of dicotyledonous angiosperms. Genome 42: 9-19.

WERNECK, F. P., G. C. COSTA, G. R. COLLI, D. E. PRADO \& J. W. SITES. 2011. Revisiting the historical distribution of Seasonally Dry Tropical Forests: new insights based on palaeodistribution modelling and palynological evidence. Glob. Ecol. Biogeogr. 20: 272-288.

WOLFE, K. H., W. H. LI, \& P. M. SHARP. 1987. Rates of nucleotide substitution vary greatly among plant mitochondrial, chloroplast, and nuclear DNAs. Proc. Natl. Acad. Sci. USA 84: 9054-9058.

WRIGHT, S. 1965. The interpretation of population structure by F-statistics with special regard to systems of mating. Evolution 19: 395-420

ZHAN, Q. Q., J. F. WANG, X. GONG \& H. PENG. 2011. Patterns of chloroplast DNA variation in Cycas debaoensis (Cycadaceae): conservation implications. Conserv. Genet. 12: 959-970.

ZHAO, Y. J. \& X. GONG. 2012. Genetic structure of the endagered Leucomeris decora (Asteraceae) in China inferred from chloroplast and nuclear DNA markers. Conserv. Genet. 13: 271-281.

Recibido el 18 de octubre de 2013, aceptado el 30 de abril de 2014 . 
